\title{
PRECISION ANALYSIS OF TRIMBLE RTX SURVEYING TECHNOLOGY WITH XFILL FUNCTION IN THE CONTEXT OF OBTAINED CONVERSION OBSERVATIONS
}

\author{
Robert Krzyżek \\ AGH University of Science and Technology, \\ Faculty of Mining Surveying and Environmental Engineering, \\ Department of Geomatics \\ Krakow, Poland
}

\begin{abstract}
As a result of traditional geodetic surveying we usually achieve observations which are then used for calculating rectangular coordinates onto a plane along with precision evaluation. In this article the surveying methods are presented in which the situation is different. Test measurements were carried out, consisting in the measurement of a fragment of detailed control network in RTK (Real Time Kinematic) and RTX (Real Time Extended) mode with xFill function. First, the rectangular coordinates onto a plane (through the transformation of data ellipsoidal) were obtained, on the basis of which the conversion observations were determined and they were compared with each other, as well as with reference parameters - conversion observations out of detailed control network adjustment with use of the method of least squares. The results of the study allow to verify the precision and application possibilities of conversion observations obtained thanks to Trimble RTX technology with $x$ Fill function. Application of this surveying method in typical geodetic tasks is fully justifiable. Nevertheless, it is recommendable to be aware of the correlations of absolute or relative values obtained in RTX procedure to reference parameters, which in turn will enable conclusive verification of the possibilities of Trimble RTX technology application in certain geodetic surveys.
\end{abstract}

Keywords: RTX, RTK GPS, conversion observations, Trimble xFill,

\section{Introduction}

The progress in information technologies, which for many years have been gradually developing in geodesy, contributed to the improvement of modern real time surveying technique RTK GPS (Real Time Kinematic Global Positioning System). This 
innovative surveying method is RTX (Real Time eXtended) technology, which is used for centimetre precision positioning of points through CenterPoint RTX service.

The measurements carried out in real time with use of RTK GPS technology enable to determine almost immediately the antenna phase centre of GPS receiver at precision level of $\pm 0.03 \mathrm{~m}$. Such measurements can be achieved thanks to OTF (OnThe-Fly) initialisation. It consists in solving the problem of phase measurement ambiguity in the rover based on the corrections sent telemetrically from the reference station, which can either be an individual physical permanent station or virtual VRS (Virtual Reference System) station, generating the data from the receiver network. The ambiguity value is most often determined with use of Kalman filter method or LAMBDA method (in new generation receivers), while the time of convergence in good conditions is approx. 10 seconds.

As each measurement method, also RTK GPS technology has some disadvantages. Apart from the necessity to carry out measurements in the open horizon terrain, there is also a problem of required permanent connectivity between the rover and the network of reference stations. In a situation when the corrections to the rover are sent through mobile network, and the rover is placed in the area of low or full outage of the provider network, then the immediate disconnection between the user rover and the reference stations occurs. The rover enters into stand-alone mode. Solution to these problems can be found in the option of PPP (Precise Point Positioning), as an alternative technique to permanent stations based on RTK measurements, namely Trimble CenterPoint RTX with xFill function. In case of the breaks in correction stream delivery from reference stations to the rover, innovative algorithms are put into operation, determining the corrections to GNSS rovers and forms of their transmission. Positioning precision is at the level of $1-2 \mathrm{~cm}$ for horizontal coordinates and $2-4 \mathrm{~cm}$ for the height. The functioning of the whole system has been described in a very detailed way in (Leonardo et al., 2011). For the purpose of this article, the operation rule of Trimble RTX technology with xFill function has been presented in modified range. Figure 1 shows distribution of about 100 control stations of CenterPoint RTX service across the globe.

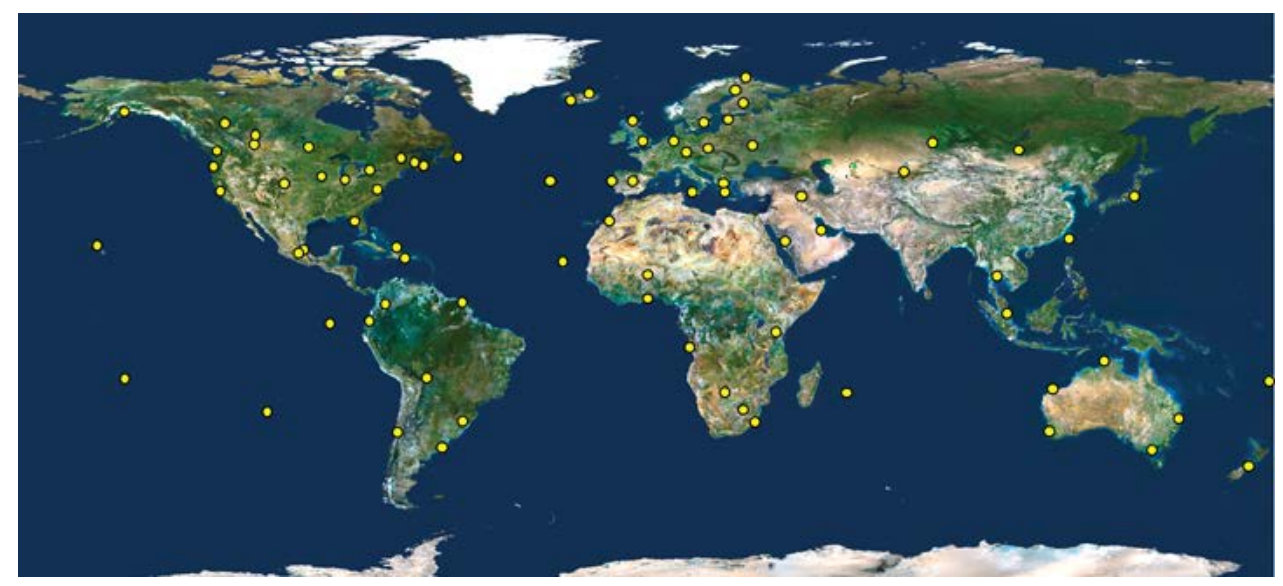

Fig. 1. Diagram of CenterPoint RTX control stations distribution (Leonardo et al., 2011)

Initially, CenterPoint RTX service was available in North and South America, by means of satellite connection. Currently corrections generated by Center Point RTX are offered in two parallel ways: via the Internet and via geostationary satellites in a special L-band Satellite. 
The process of Trimble RTX system operation with xFill function can be presented in a simplified manner in a few points:

1. The operation of rover $R$ in typical field conditions: receiving correction streams from the individual physical reference station $B$ or virtual station VRS (fig. 2).

2. Difficult field conditions resulting in signal outage, received from the reference station by the user rover (Fig. 3).

3. At the same time, all information from satellites is collected by RTX system control stations and then sent via Internet to the so called operative centres (Fig. 3).

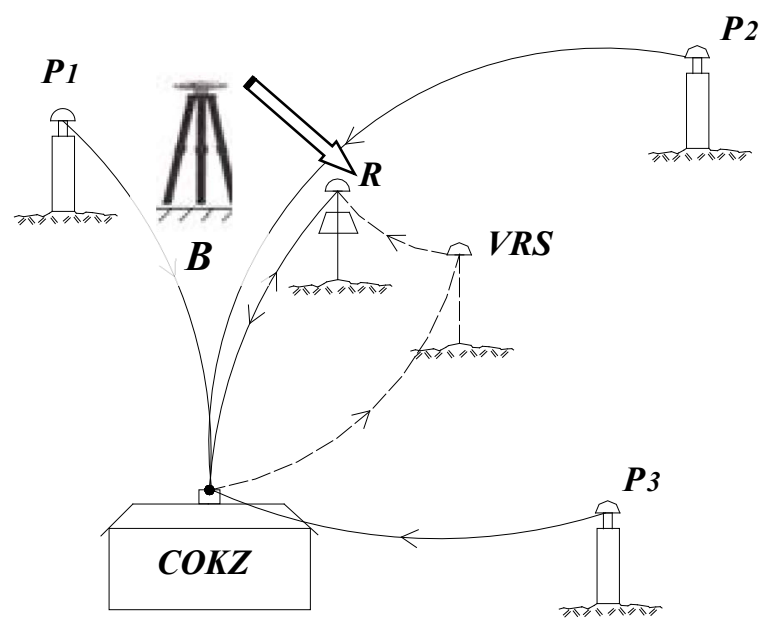

\author{
VRS - Virtual Reference Station \\ Correction \\ $\boldsymbol{B}$ - Physical Reference Station \\ Correction \\ $\boldsymbol{R}$ - User Rover \\ $\boldsymbol{P}_{\boldsymbol{i}}-$ Reference Station \\ COKZ - The Calculation Control \\ and Management
}

Fig. 2. Alternative correction sources for RTK measurements:

individual physical reference station $B$ or diagram of virtual reference station VRS.

In the operative centres there are additional communication servers, which play an important role in functioning of the system. Their task is to process the satellite data (precise satellite orbit, satellite clock or observation predictability) and passing them to further information flow. Then, after generating precise satellite information, its compression takes place, compliant with CMRx format. Further flow of information, included in the compressed message, can be carried out in two ways. The first way of making the message available to all users is done via Internet. The second option is to route the message via uplink station to the satellite, and then referring the data directly to the recipient.

Such solution makes it possible to perform work in real time by the rover in permanent way, even when it encounters the area of signal outage. In such situation, the user can observe on the controller screen (Trimble R10 GNSS receiver) the automatic switch from standard RTK mode with the so called ambiguity-fixed solution mode into RTX mode with xFill function, along with the changing values of horizontal errors $\mathrm{H}$ and vertical errors $\mathrm{V}$ (Fig. 4). 


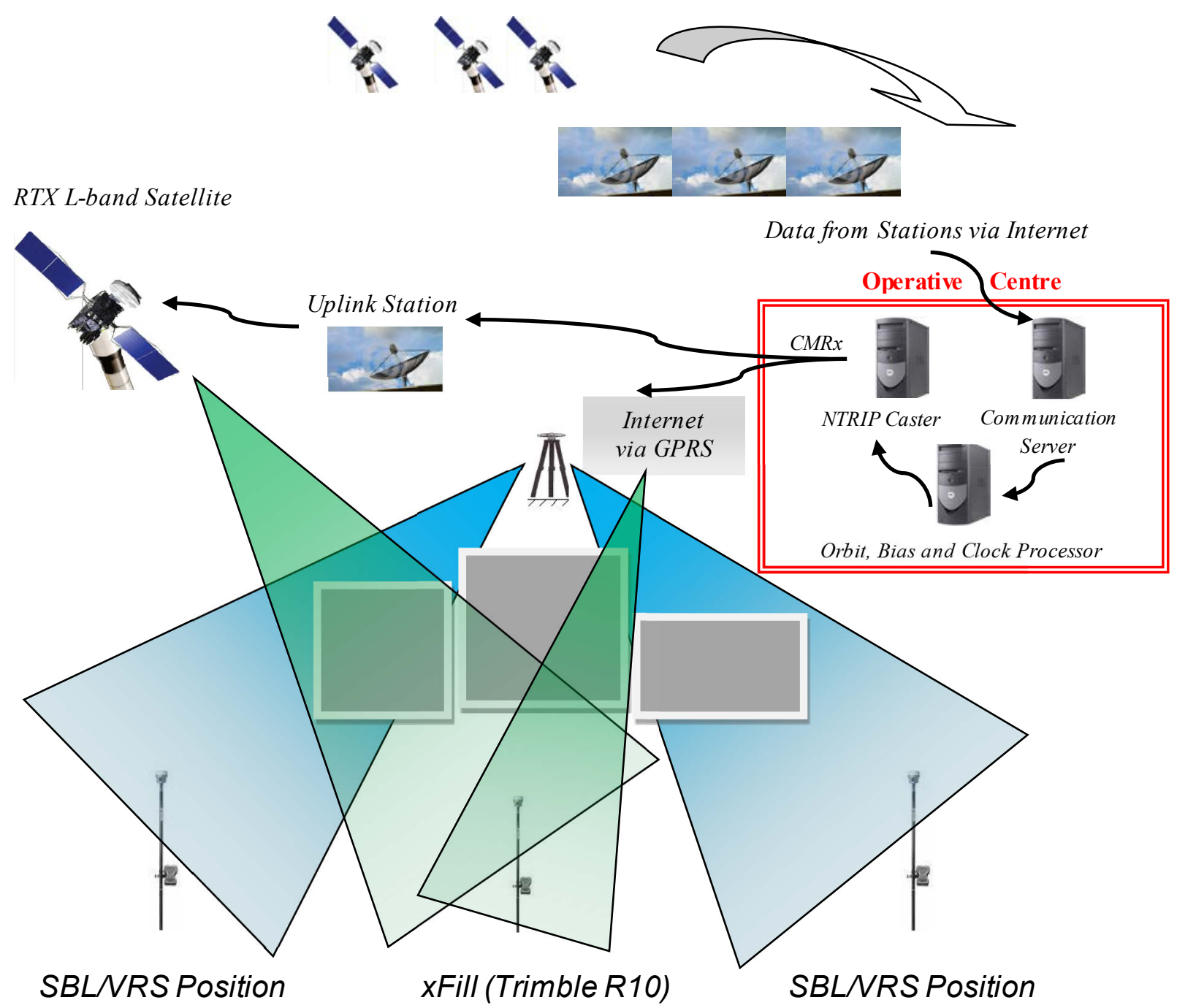

Fig. 3. General infrastructure of RTX technology system functioning along with the simultaneous reaction of the rover with use of Trimble xFill function.

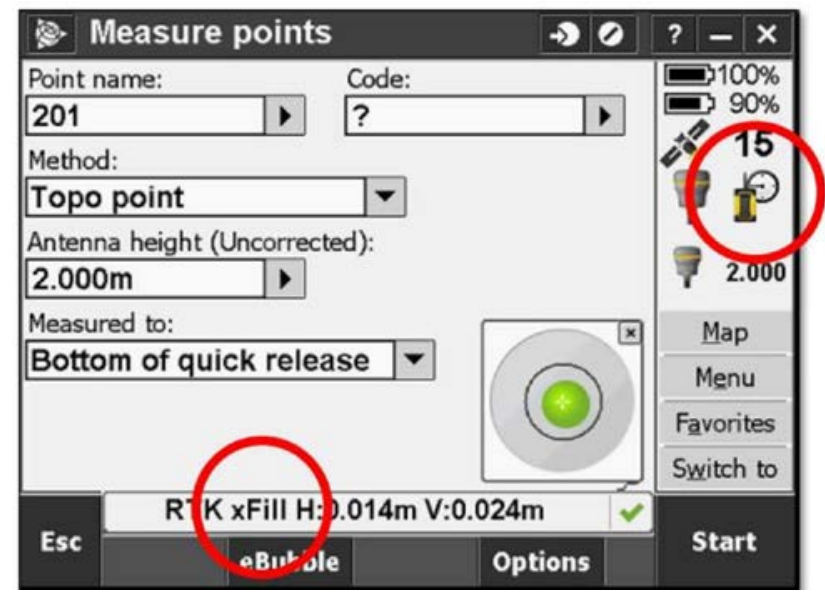

Fig. 4. Dialogue box of Trimble R10 GNSS receiver after switching to RTX mode with xFill function. 
The process enables ambiguity weighting, which in turn enables better estimation of errors in relation to traditional GNSS solutions. Continuation of operation in real time in RTX mode with XFill function is limited in time, which can also be monitored in the controller screen of the mentioned GNSS receiver (Fig. 4). According to the manufacturer information, this time is limited to 5 minutes.

An interesting way of solving the problem of ambiguity was presented in the studies by (Kubo, 2009). They showed the benefits of adding speed information to the positioning in RTK mode. The study was conducted in suburban areas, where potentially the decline of signal received by the rover may occur. The measurement of Doppler frequency speed was used to solve the ambiguity with LAMBDA method. They also proved that Doppler frequency measurements do not adversely affect in a significant way the pseudo distance measurement, because multipath phenomenon occurring in Doppler frequency measurements is at the level of individual centimetres. Therefore, the proposed solution of ambiguity on individual epoch has improved the efficiency in comparison to the traditional solution of ambiguity not allowing for the speed information.

In their research experiments (Dai et al., 2003) also verified the problem of ambiguity solution, this time, however, in the context of significant delay of atmosphere residuals in double differential measurements. They discussed time and space properties of the correlation for atmospheric delay and proposed relevant models for anticipating atmospheric delays. Experiment results show that the proposed models for anticipating delays can be efficient and securely used for solving ambiguity in reference station network in real time, based on individual epoch.

Much emphasis on efficiency and reliability of ambiguity solution put (GrejnerBrzezieńska et al., 2007). They discussed four different methods of ionosphere modeling, used as a source of information, and their impact on the speed and reliability of ambiguity solution and the rover positioning accuracy.

As it was mentioned before, Trimble RTX technology with xFill function operates on the basis of precise satellite information -satellite orbit, satellite clock, observation predictability - generated in operation centres (Fig. 3) and transmitted to the users. The requirements in relation to satellite orbits, which are used in the global RTX system, can be defined with precision, reliability, continuity and steadiness. These elements were described in (Leonardo et al., 2011).

One of the most significant data of RTX satellite system is estimation of satellite clock errors. It is coessential with solving the problem of phase measurement ambiguity and therefore it is transmittable in a direct way to the precision of rover determination. The time being a delay in calculating errors of satellite clock definitely lowers the precision of point determination. That is why a very important factor in satellite clock structure is the way, and to be precise - the time of processing data from many reference stations of the system.

The effective way of estimating the clock errors was presented by (Zhang et al., 2011). It referred to joint use of the algorithm of double plot consisting of undifferenced (UD) and epoch-differenced (ED) engine. UD engine creates the absolute value of the clock every 5 seconds, and ED engine produces relative values of the clock between the neighbouring epochs in at 1 second intervals. As a final result, the satellite clock frequency of $1 \mathrm{~Hz}$ can be achieved through connecting UD of the clock absolute values and ED of the clock relative values.

Different solution to the problem of satellite clock errors was presented by (Lannes et al., 2013). It refers to phase delays of the clock in GNSS. They proposed solution 
to the problem through undifferenced approach based on the concept of ambiguity closure. They presented theoretical justification of closure-ambiguity approach (CAA) as well as the main elements for its practical implementation, which in turn can be an interesting solution in real time surveying, e.g. PPP. In this solution, when the phase delay is estimated for the first time, its fractional part is limited to one cycle of interval range cantered to zero; then the total ambiguity is modified accordingly.

Another satellite information, generated in operational centres of RTX system (Fig. 3 ), is observation predictability. Achieving observations at centimetre-level precision is possible only with correct modelling of this parameter. Such process enables observation continuity, which in turn allows to prevent the occurrence of inconsistent observation modelling in time.

A very important factor in the operation of Trimble RTX system with xFill function is the time needed for obtaining convergent solution. It depends on different elements that may affect the positioning precision, e.g. multipath phenomenon or satellite constellation. RTX technology uses a range of functions that reduce obtaining convergence in shortest time possible. They were described in (Leonardo et al., 2011). Final convergence of the system can be obtained within 10 to 45 minutes after the receiver starts working.

The use of two satellite systems, GPS and GLONASS, in Trimble RTX technology is an alternative solution, aimed at minimalizing convergence time. The studies carried out (Zhang et al., 2013) indicate the possibility of reducing the time of achieving convergent system solution with use of GPS and GLONASS by $42 \%$.

Other studies refer to the problem of time of achieving another or even repeated convergence of the system. The method proposed by (Geng et al., 2010) consists in precise estimation of ionospheric delay, which greatly speeds up the ambiguity solution. First of all, the ambiguity for wide-lane linear combination can be solved fast by means of wide-lane linear combination corrected by the influence of the ionosphere. Second of all, ambiguity solution for narrow-lane linear combination can be significantly speeded up by narrowing the ambiguity search, obtained from ambiguity solution in wide-lane bandwidth, corrected with ionosphere influence. As a result, the method proposed can potentially minimalize the requirements to maintain continuously open sky horizon typical for the majority of PPP applications and improve the implementation of precise point positioning in real time.

The standards of conducting geodetic measurements, in real time using RTK GPS technologies are regulated in Poland by the ordinance of the Minister of Interior and Administration of 9 November 2011 in case of technical standards of performing detailed surveys and working out and sending results of these surveys to National Geodetic and Cartographic Resource. The legal act obliges the persons who conduct the measurements to determine the so called geodetic control points (e.g. geodetic control points of detailed control network class III) using RTK technology, and then to compare the coordinates obtained with catalogue database. The maximum allowable coordinate differences from geodetic control points measurements are - $d X$, $\mathrm{dY}= \pm 0.12 \mathrm{~m}, \mathrm{dH}= \pm 0.09 \mathrm{~m}$. Such verification relates, however, to absolute values not allowing for the aspect that geodetic control points coordinates can be marked in relation to other reference parameters than these obtained through RTK measurement. The aim of this paper is to verify the precision of Trimble RTX technology with xFill function in the context of the achieved conversion observations, so the relative values from the measurement conducted in real time. This verification refers not only to mutual relations between two related measurement methods, RTK 
and RTX, but also to data (reference parameters), which could serve as geodetic control points.

\section{Research experiment}

In order to conduct research experiment the results of geodetic measurements in real time were verified on the points belonging to detailed control network class III, located in Malopolska Province in Jerzmanowice-Przeginia Commune (Poland). The research object presenting the fragment of test network is illustrated in Fig. 5 . On the basis of the geodetic and cartographic documentation kept by National Geodetic and Cartographic Resource the precision of marking location of the mentioned geodetic network control points on the given object was determined. The results of strict adjustment of geodetic control network points presented in geodetic selection range are shaping an average positioning error of these points $\left(M_{P}\right)$ not larger than \pm 0.003 $\mathrm{m}$ and $\pm 0.005 \mathrm{~m}$ for the height $\left(M_{H}\right)$. All measurable or conversion values conducted on the test object during the research experiment were related to parameters determined on the basis of geodetic control network point coordinates, the source of which was the adjustment of the whole network using the method of least squares. This way the parameters became reference elements for further results of research measurement. It should be definitely emphasized, however, that the parameters mentioned, or their source data, are not error-free values. The correctness of this supposition results from the fact that in the research experiment precision of the measurement technology itself is verified in the context of certain assumptions, and generally assumed measurement precision of the technology in real time is at ten times lower level than the obtained average errors of geodetic control network points from strict adjustment.

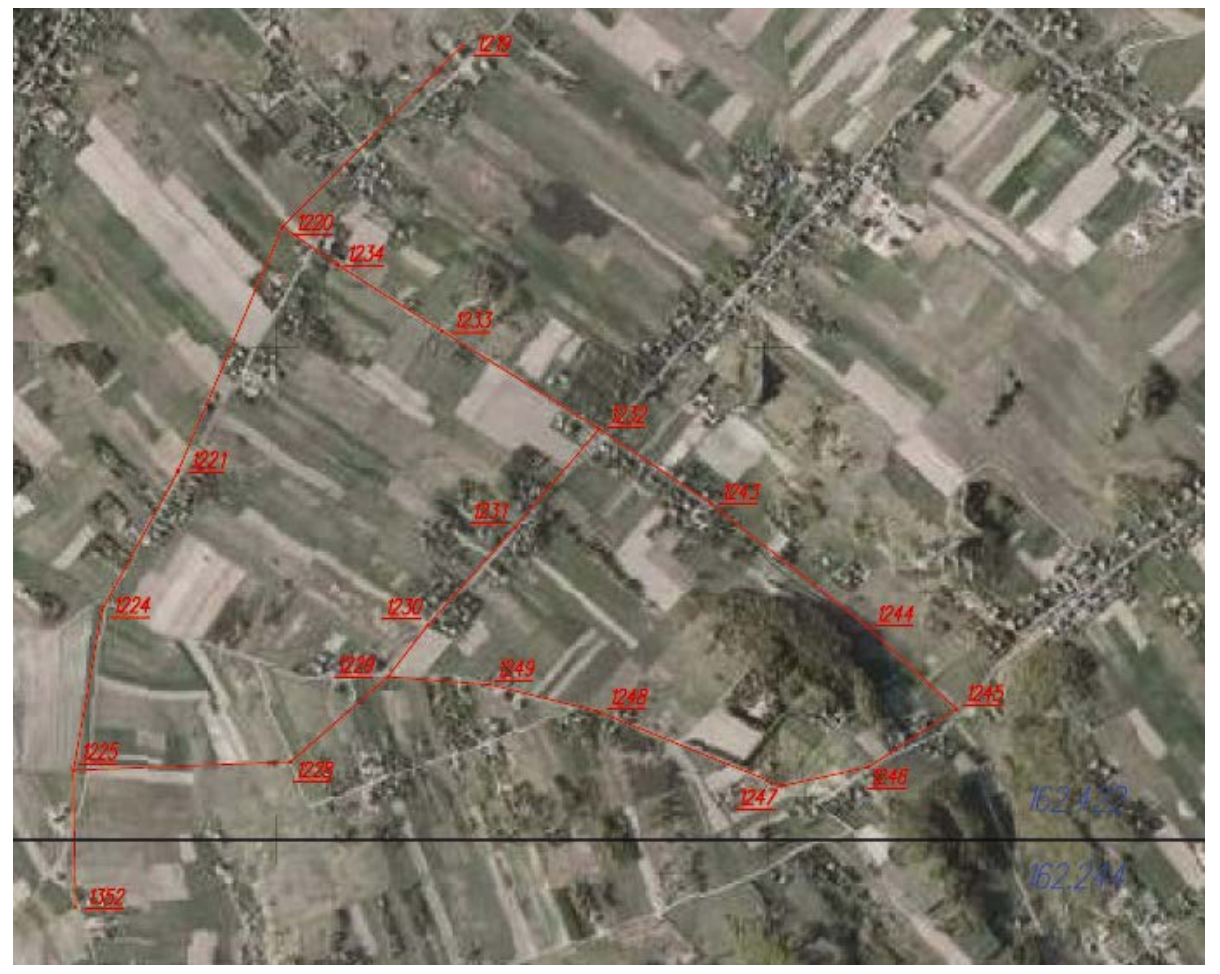

Fig. 5. The outline of detailed control network class III (test control network) on the object of „Jerzmanowice-Przeginia” 
Each control network point was measured using real time technology in two modes: RTK (Real Time Kinematic) and RTX (Real Time Extended) using xFill function. Switching to RTX mode took place immediately after the measurement in RTK GPS was finished. In order to conduct the research experiment, Trimble R10 GNSS receiver was used in relation to ASG EUPOS reference station network.

Since 2008, the network of permanent geodetic stations ASG EUPOS is in operation in Poland. The points of these stations constitute the basic geodetic control network in Poland and are evenly distributed in the whole area (Fig. 6). The existing EPN stations (EUREF Permanent Network) as well as IGS stations (International GNSS Service) were also included into the network of reference stations.

Their importance in the realisation of reference to local GPS networks is described by (Bosy et al., 2009). Distribution of ASG EUPOS reference stations (the average distance between stations is $70 \mathrm{~km}$ ) enables to conduct measurements in RTK mode in each location with precision required. The system of permanent stations makes the following real time services available to the users: NAWGEO, KODGIS and NAWGIS. For the research experiment, during the measurements in RTK and RTX mode, NAWGEO_VRS_2_3 service was used. Fig. 6 shows also the location where test measurements were conducted, accompanied by the nearest stations of ASG EUPOS system.

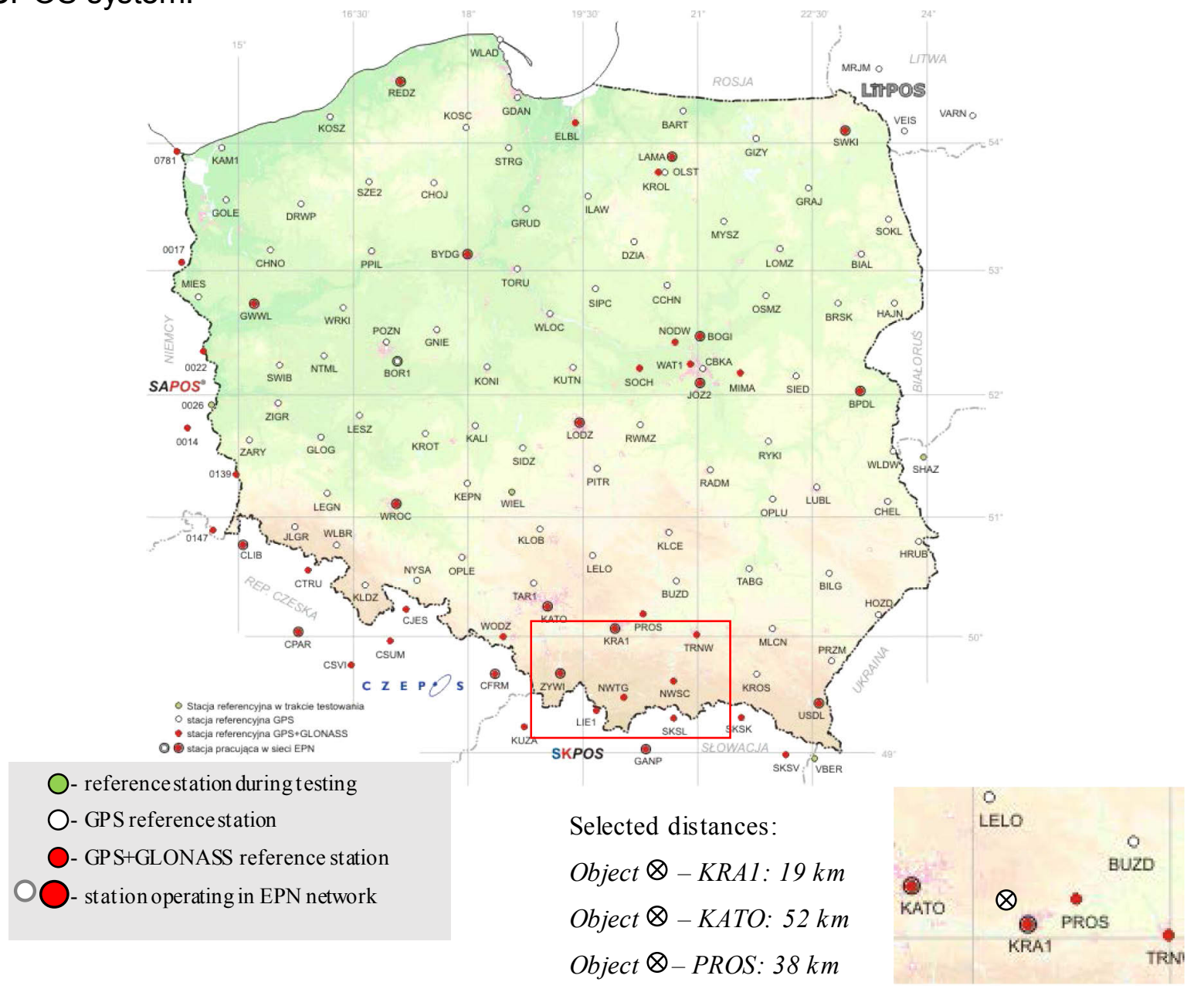

Fig. 6. Distribution of ASG-EUPOS reference stations in Poland along with the location of research object (www.asgeupos.pl, 2013) 
Conducting the measurements of test object in RTK and RTX mode using xFill function the rectangular coordinates onto a plane $\mathrm{X}, \mathrm{Y}$ and height $\mathrm{H}$ were obtained in the national system PL 2000. Due to the character of test points - geodetic detailed control network - the measurement results for RTK GPS mode were the average value from 30 measurement epochs. In case of Trimble RTX technology the measurement was released manually every few or over a dozen seconds.

In Trimble R10 GNSS receiver, which enables the use of a few interesting innovative solutions in geodetic measurements, so called SurePoint technology was activated. It ensures recording measurement results only within specified (established) limits of measurement pole vertical deflection. Parameter of the pole vertical deflection was defined at the value of $\pm 0.010 \mathrm{~m}$. The manufacturer of GNSS receiver assures also that it is possible to continue measurements for 5 minutes after connection outage with reference stations and switching into RTX mode with xFill function. This time period has been verified during research experiment in (Krzyżek, 2013).

As it was mentioned before, the control network points of the test object were measured in RTK GPS and RTX mode wit xFill function, as a result of which the rectangular coordinates onto a plane $X, Y$ and height $H$ were obtained. Detailed comparison of mutual relations between individual measurement methods for the coordinate values achieved was discussed in (Krzyżek, 2013). In this paper, however, the conversion observations based on the coordinates achieved were analysed. Based on coordinates achieved in RTK GPS and RTX mode wit xFill function, calculation of typical geodetic observations was conducted, such as distances, angles or height differences between the neighbouring points of control network, as well as intermediary values in form of control angle sums and area fields in "loops". Comparative analysis referred to combinations of individual methods: RTK - RTX, RTK - KAT., RTX - KAT. (KAT. relates to conversion observations established as reference parameters). As a result of traditional geodetic surveying we usually achieve observations which are then used for calculating coordinates along with precision evaluation. In the study methods used we are dealing with different situation. First, rectangular coordinates were obtained, on the basis of which observation calculation and mutual comparison was carried out. This comparison resulted in the differences of the analysed parameter for individual combinations. In reference to achieved in this way parameter differences null hypothesis $H_{0}$ was assumed, which reads as follows: mean value $\mu$, for individual parameters differences $(d D, d \alpha, d h, d K, d P)$ in respective combinations of methods (RTK-RTX; $R T K-K A T$.; RTX-KAT.), is equal to the established value $\mu_{0}=0$.

$$
H_{0}: \mu=\mu_{0}
$$

For each null hypothesis, an alternative hypothesis has to be specified, which for this study has been defined as follows: mean value $\mu$, for individual parameters differences ( $d D, d \alpha, d h, d K, d P)$ in respective combinations of methods (RTK-RTX; RTK-KAT.; RTX-KAT.), is not equal to the established value $\mu_{0} \neq 0$.

$$
H_{1}: \mu \neq \mu_{0}
$$

For each type of calculation-based observations compared in the individual methods, the average value of $\mu$ referred to the differences in these observations $(d D, d \alpha, d h$, $\mathrm{dK}, \mathrm{dP}$ ). Due to an insignificant number of the analyzed test points, the $T$-test model 
of a single mean value was used for the statistical analysis, which was expressed by the formula:

$$
T=\frac{\mu-\mu_{0}}{\sigma(\mu)}
$$

where:

$\sigma$ - average value of standard deviation expressed by the formula:

$$
\sigma(\mu)=\frac{\hat{\delta}}{\sqrt{n}}
$$

where:

$\hat{\delta}$ - standard deviation for parameter differences $(d D, d \alpha, d h, d K, d P)$ in respective combinations of methods,

$\mathrm{n}$ - number of parameters $(d D, d \alpha, d h, d K, d P)$ in respective combinations of methods.

Variable $T$ of the applied test model of an individual mean value has the distribution T-Student for the assumed significance level $\alpha=5 \%$ on $k=n-1$ degrees of freedom. Based on such distribution, two sided critical region was constructed, allowing for $T$-Student distribution quintile $t(\alpha, k)$.

At the beginning of research experiment description, the origin of reference parameters was determined, meaning conversion observations obtained on the basis of control network point coordinates from adjustment using the method of least squares. Location of those points was determined using polygonization method in relation to tie in points, which in turn may account for the local character of reference points. Location of the same points of test object network determined in RTK mode is strictly related to the network coordinates of ASG EUPOS station. Therefore, it would be recommendable first to analyse homogeneity of research sample in respective combinations. That is why the randomly selected relative values were subject to analysis - increments of horizontal coordinates, distance and height differences - between each network point (out of methods: RTK, RTX, KAT.) and the nearest permanent ASG EUPOS station, meaning station KRA1.

In reference to the way of conducting statistical analysis presented before, the compilation of parameters mentioned was made (relative values in relation to stations KRA1), calculated based on the rectangular coordinates onto a plane, and presented in tables 1-3. What is more, in figures 7-11 the histograms were illustrated, presenting the occurrence frequency of the calculated differences of conversion observations.

In table 1 the measurement results were verified in the context of calculated increments of horizontal coordinates. The shadowed cells in Table 1 (and in the other tables 2 - 4 as well) indicate observation exclusion from the analysis. It is due to the fact of damage to the point no 1243. The results included, along with their statistical analysis for increment differences $D d X$ explicitly present lack of grounds to reject hypothesis $H_{0}$, but only for combination of real time RTK-RTX methods. In other two comparisons (RTK-KAT. and RTX-KAT.) the conclusion prevails of rejecting hypothesis $H_{0}$ for the benefit of hypothesis $H_{1}$. Taking into account the study results 
included in (Krzyżek, 2013) and some kind of precision predictability of respective measurement methods, the results from Table 1 for the increment differences $D d X$ can be deemed right and understandable.

Some more doubts, however, can relate to statistical analysis results for increment differences $D d Y$ (Table 1). Although for the comparison of methods RTK-RTX. there are also no grounds for rejection of the hypothesis $H_{0}$, the same conclusion, however, can be reached for the results comparison of RTX-KAT. method. Analysing more carefully the results included in Table 1 for methods RTK-KAT (with the conclusion of rejecting hypothesis $H_{0}$ for the benefit of hypothesis $H_{1}$ ) and RTX-KAT., a generally high convergence of the results achieved can be noticed. If we considered the variable $T$ of the applied test model of individual mean value for the assumed significance level $\alpha=10 \%$ at $k=n-1$ degrees of freedom, then both for the combination of methods RTK-KAT. and RTX-KAT. there would be a common conclusion about rejecting hypothesis $\mathrm{HO}$ for the benefit of hypothesis $H_{1}$. 


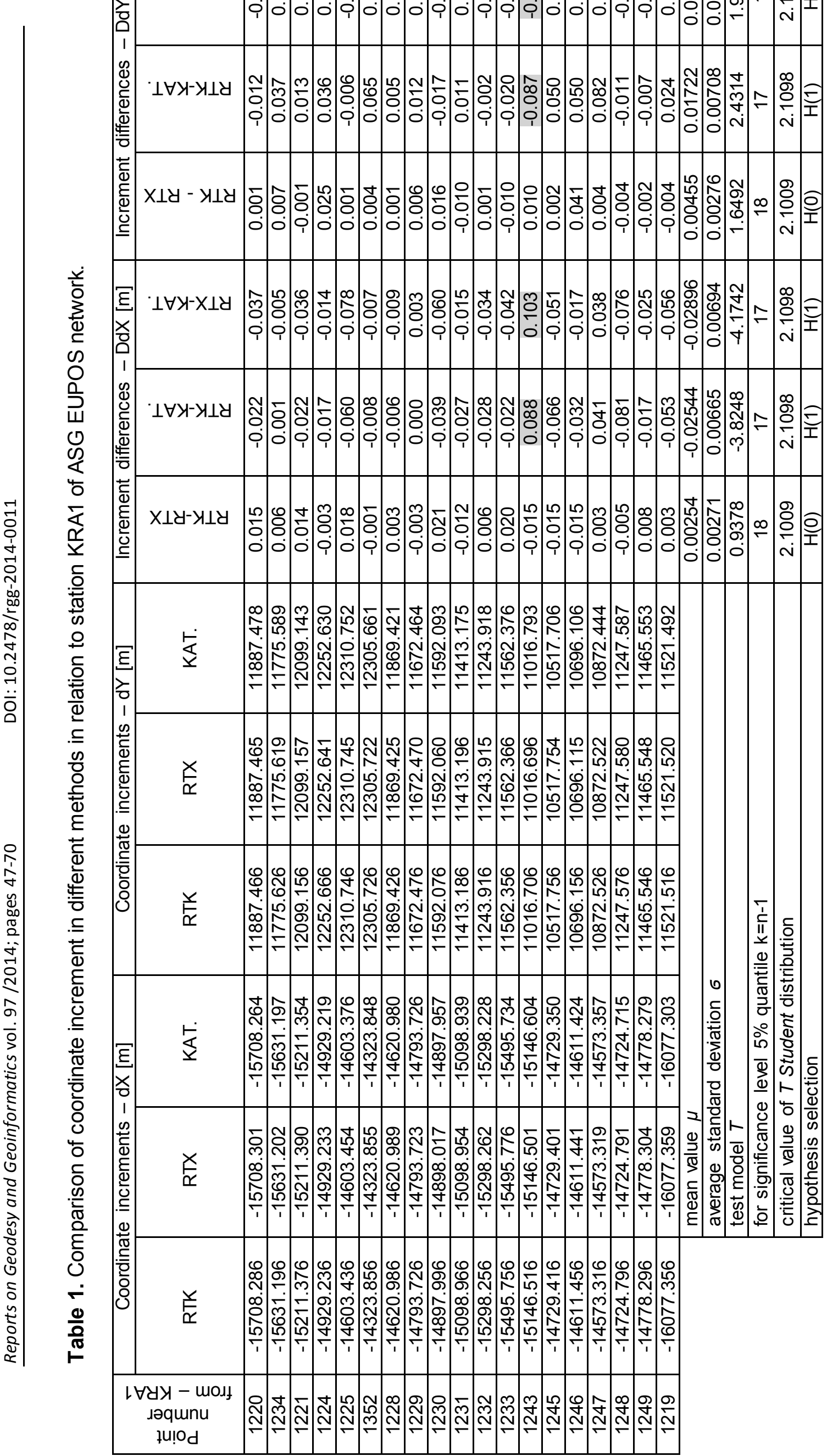

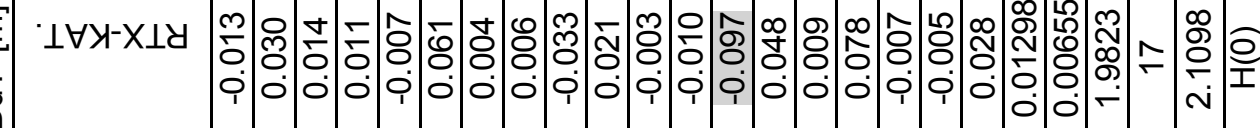

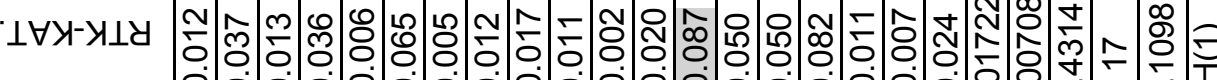

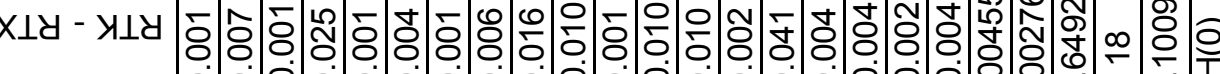

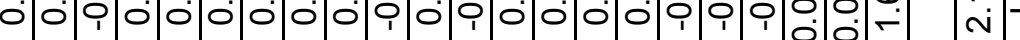




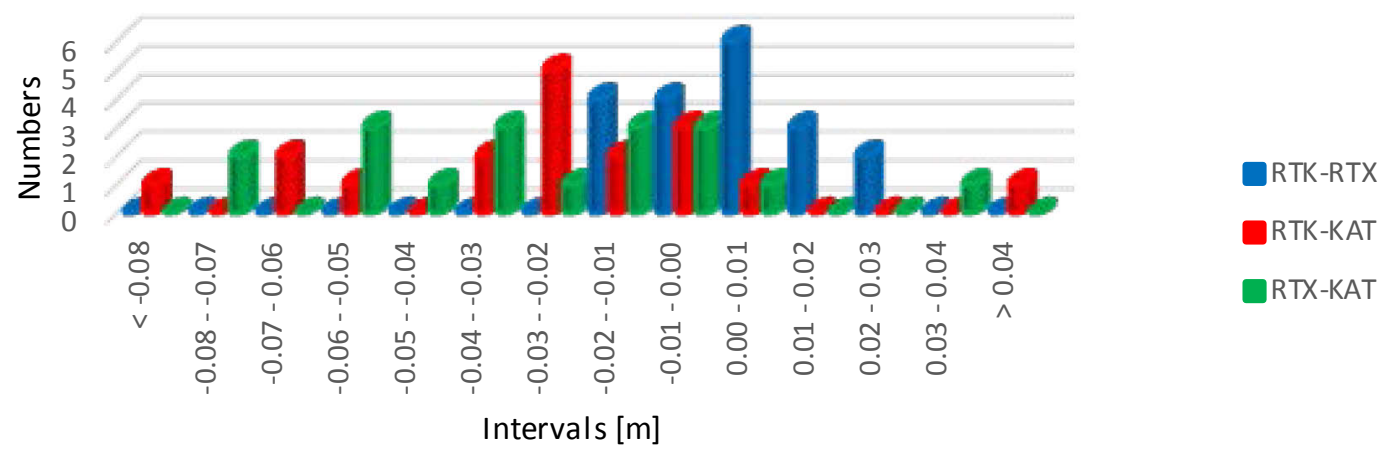

Fig. 7. Histogram of the frequency measured for coordinate increment differences $D d X$ between the methods: RTK-RTX; RTK-KAT.; RTX-KAT. in relation to station KRA1 of ASG EUPOS network.

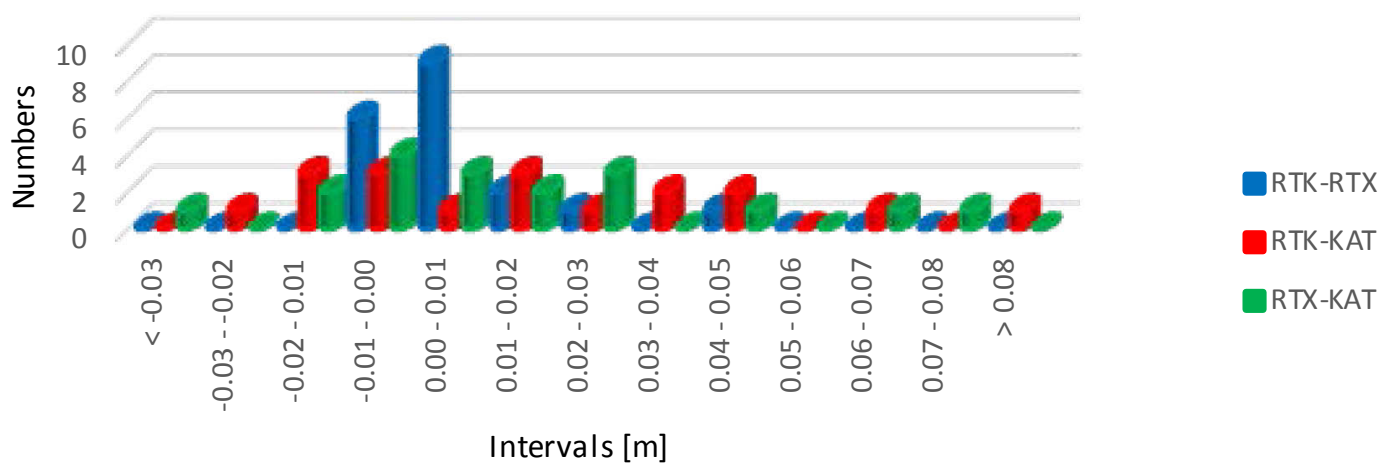

Fig. 8. Histogram of the frequency measured for coordinate increment differences $D d Y$ between the methods: RTK-RTX; RTK-KAT.; RTX-KAT. in relation to station KRA1 of ASG EUPOS network.

Figures 7 and 8 presenting the histograms of frequency measured for both coordinate increments $D d X$ and $D d Y$ in combination of methods RTK-RTX accumulate the highest number of compared parameters occurrence within the range of $-0.02 \mathrm{~m}$. do $+0.02 \mathrm{~m}$. for increment differences $D d X$ and of $-0.01 \mathrm{~m}$. do $+0.01 \mathrm{~m}$. for increment differences $D d Y$. Thus, these are small values, staying within the limits of measurement precision in RTK or RTX mode. For the combination of other methods (RTK-KAT. and RTX-KAT.) the ranges of measured frequency occurrence increase significantly, which only confirms in this case the rightness of the conclusion about rejecting hypothesis $\mathrm{HO}$ for the benefit of hypothesis $\mathrm{H} 1$ rejecting hypothesis $\mathrm{H}_{0}$ for the benefit of hypothesis $H_{1}$.

Another comparison that is subject to statistical analysis are distances (Table 2 and Fig. 9) and elevations (Table 3 and Fig. 10) between each network point (out of methods: RTK, RTX, KAT.) and the reference station KRA1. In case of both mentioned parameters the final conclusions are similar and are nearly the continuation of the conclusions of horizontal coordinate increment comparison. Therefore, for the relation of methods RTK-RTX there are no grounds for rejecting the hypothesis $H_{0}$,, while in other cases (RTK-KAT. and RTX-KAT.) the conclusion prevails of rejecting hypothesis $H_{0}$ for the benefit of hypothesis $H_{1}$. 
Table 2. Comparison of distances in different methods in relation to station KRA1 of ASG EUPOS network.

\begin{tabular}{|c|c|c|c|c|c|c|}
\hline \multirow{2}{*}{ 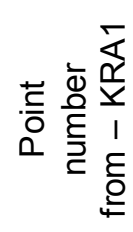 } & \multicolumn{3}{|c|}{ Distance - D [m] } & \multicolumn{3}{|c|}{ Distance differences $-\mathrm{dD}[\mathrm{m}]$} \\
\hline & RTK & RTX & KAT. & RTK-RTX & RTK-KAT. & RTX-KAT. \\
\hline 1220 & 19699.292 & 19699.303 & 19699.281 & -0.012 & 0.010 & 0.022 \\
\hline 1234 & 19570.377 & 19570.378 & 19570.356 & -0.001 & 0.021 & 0.022 \\
\hline 1221 & 19436.449 & 19436.460 & 19436.423 & -0.012 & 0.025 & 0.037 \\
\hline 1224 & 19313.465 & 19313.446 & 19313.429 & 0.018 & 0.036 & 0.018 \\
\hline 1225 & 19100.126 & 19100.139 & 19100.084 & -0.013 & 0.042 & 0.055 \\
\hline 1352 & 18883.955 & 18883.951 & 18883.906 & 0.004 & 0.048 & 0.045 \\
\hline 1228 & 18832.326 & 18832.328 & 18832.318 & -0.002 & 0.008 & 0.010 \\
\hline 1229 & 18844.124 & 18844.119 & 18844.117 & 0.006 & 0.007 & 0.002 \\
\hline 1230 & 18876.613 & 18876.619 & 18876.592 & -0.007 & 0.020 & 0.027 \\
\hline 1231 & 18927.218 & 18927.215 & 18927.190 & 0.003 & 0.028 & 0.025 \\
\hline 1232 & 18985.844 & 18985.849 & 18985.823 & -0.004 & 0.021 & 0.026 \\
\hline 1233 & 19334.077 & 19334.099 & 19334.071 & -0.022 & 0.006 & 0.028 \\
\hline 1243 & 18729.249 & 18729.231 & 18729.371 & 0.018 & -0.122 & -0.140 \\
\hline 1245 & 18099.141 & 18099.127 & 18099.058 & 0.013 & 0.083 & 0.069 \\
\hline 1246 & 18108.076 & 18108.039 & 18108.020 & 0.037 & 0.055 & 0.019 \\
\hline 1247 & 18182.227 & 18182.226 & 18182.210 & 0.000 & 0.016 & 0.016 \\
\hline 1248 & 18529.101 & 18529.100 & 18529.043 & 0.001 & 0.058 & 0.056 \\
\hline 1249 & 18704.459 & 18704.467 & 18704.450 & -0.008 & 0.009 & 0.017 \\
\hline \multirow[t]{7}{*}{1219} & 19779.452 & 19779.456 & 19779.395 & -0.004 & 0.057 & 0.062 \\
\hline & \multicolumn{3}{|c|}{ mean value $\mu$} & 0.0008 & 0.0307 & 0.0308 \\
\hline & \multicolumn{3}{|c|}{$\begin{array}{l}\text { average standard deviation } \sigma \\
\text { test model } T\end{array}$} & 0.0031 & 0.0052 & 0.0045 \\
\hline & \multicolumn{3}{|c|}{ test model $T$} & 0.2635 & 5.8895 & 6.8644 \\
\hline & \multicolumn{3}{|c|}{ for significance level $5 \%$ quantile $k=n-1$} & 18 & 17 & 17 \\
\hline & \multicolumn{3}{|c|}{ critical value of $T$ Student distribution } & 2.1009 & 2.1098 & 2.1098 \\
\hline & \multicolumn{3}{|c|}{ hypothesis selection } & $\mathrm{H}(0)$ & $H(1)$ & $\mathrm{H}(1)$ \\
\hline
\end{tabular}

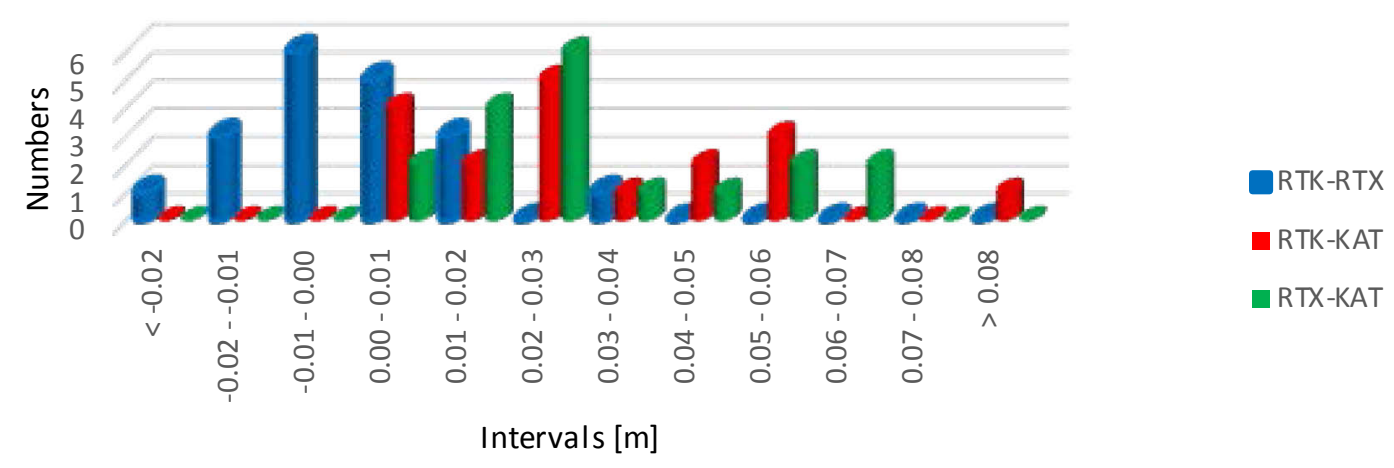

Fig. 9 Histogram of the frequency measured for distance differences $d D$ between the methods: RTK-RTX; RTK-KAT.; RTX-KAT. in relation to station KRA1 of ASG EUPOS network.

Analysing relations between test model $T$ of individual mean value and critical values of $T$ Student distribution (Table 2 and 3 ), not only the conclusion about the selection of a given hypothesis, presented above, can be reached. This comparison shows also a wide span of calculations achieved, which only strengthens the conclusions which verify selection of the hypothesis. It refers especially to the results in Table 2 for all comparative methods and the results from Table 3 for RTK-RTX 
relation combination. Slightly smaller divergence of the calculations achieved refers to the combination RTK-KAT. and RTX-KAT. (Table 3), however, still sufficient to reject hypothesis $H_{0}$ for the benefit of hypothesis $H_{1}$.

Table 3. Elevation comparison in different methods in relation to station KRA1 of ASG EUPOS network.

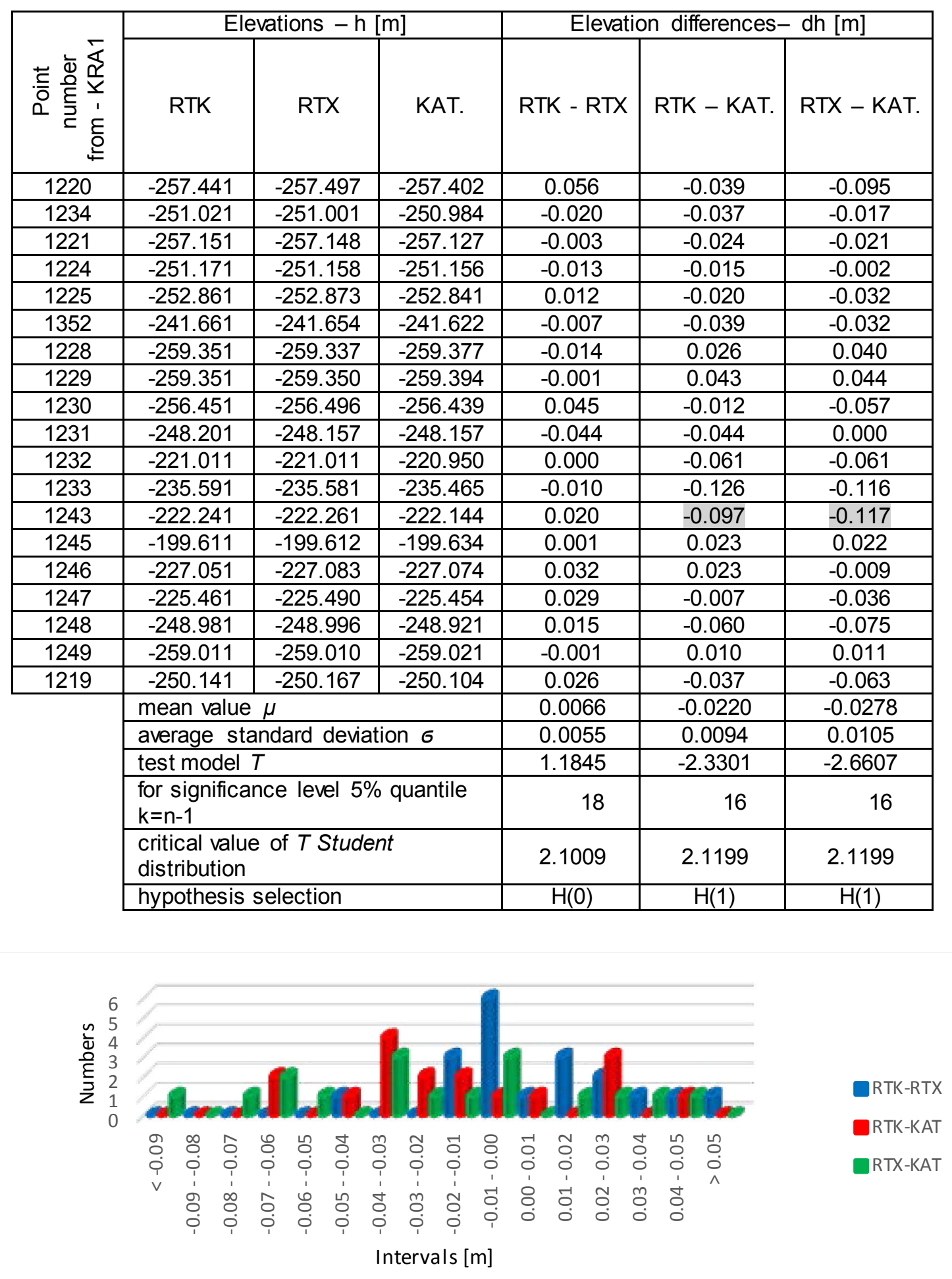

Fig. 10 Histogram of the frequency measured for elevations $h$ between the methods: RTK-RTX; RTK-KAT.; RTX-KAT. in relation to station KRA1 of ASG EUPOS network. 
When we look at the measured frequency of analysed conversion observations (Fig. 9 and 10), we can see the relations of methods RTK-RTX, which for distance concentrate mainly within the range of $-0.02 \mathrm{~m}$. to $+0.02 \mathrm{~m}$. (Fig. 9), and for elevations from $-0.02 \mathrm{~m}$. to $+0.03 \mathrm{~m}$. (Fig. 10). Taking into consideration the fact that we are dealing with real time measurement technology, we can assume that the values within these ranges are small, or even ignorable. The ranges for other method relations (RTK-KAT. and RTX-KAT.), both for distance (usually from $0.00 \mathrm{~m}$. to + $0.07 \mathrm{~m}$.) and for elevations (usually from $-0.07 \mathrm{~m}$. to $+0.06 \mathrm{~m}$.), have much higher values, and therefore they grant variable character upon the analysed parameters.

If we were to finish the analysis of hitherto existing parameters at the current stage, it should be concluded that verification of conversion observations in method relations RTK-RTX is convergent, and therefore both methods can complement each other, at the same time retaining the required precision of the measurement results achieved. It would be a mistake, on the other hand, to use conversion observations in method relations RTK or RTX for the assumed method KAT. Reference parameters, in relation to which the coordinates were determined and then the conversion observations of test network points for the method denoted KAT. are to a significant extent different with regards to methods of real time measurement RTK and RTX. That is why the conclusion relating to the test on homogeneity of a test sample proved to be negative.

Despite negative verification of test sample homogeneity and the conclusions reached, the comparison of conversion observations was carried out, in relation to similar parameters, however, this time between individual control network points of the test object. The aim of this experiment was to evaluate how the test area size affects the final conclusions reached.

First combination referred to distance (Table 4). All distances (out of methods: RTK, RTX, KAT.) were calculated based on the rectangular coordinates obtained. Coordinates for methods RTK and RTX were obtained directly from the measurement, while for method KAT. they were obtained from the adjustment of control network points using the method of least squares.

The results included in Table 4 clearly indicate lack of grounds for rejecting hypothesis $H_{0}$. Mutual relation between the values of test model $T$ and critical values of T-Student distribution additionally allow to strengthen the conclusion about lack of grounds to reject hypothesis $H_{0}$. Analysing individual mutual spans of distance differences in respective combinations of methods we can observe their upward trend. For method RTK-RTX they oscillate in the range of $-0.033 \mathrm{~m}$ to $+0.041 \mathrm{~m}$, for the relation RTK-KAT. this range is increasing from $-0.050 \mathrm{~m}$ to $+0.051 \mathrm{~m}$ and for the last comparison RTX-KAT. it reaches the level of $-0.069 \mathrm{~m}$ do $+0.079 \mathrm{~m}$. In order to better visualise the occurrence of so called frequency measured in respective combinations of methods, the number of respective distance differences has been illustrated for the ranges established (Fig. 11).

Based on data included in Table 4 and Figure 11 high convergence can be noticed of comparative results obtained (distance differences) between methods RTK-RTX. Generally, these are differences at the level of a few or a dozen or so millimetres. For two sides only, that is 1245-1246 and 1246-1247, they are at the level of $\pm 0.03 \mathrm{~m}$ and only in one case $(1230-1231)$ they exceed $\pm 0.04 \mathrm{~m}$. It can be definitely assumed then that distance conversion observations - for the measurement in RTK and RTX mode are on the same level of precision. When we conduct verification of distance differences in other combinations (RTK-KAT., RTX-KAT.), the tendency of higher value ranges occurrence for conversion observation can be firmly observed. 
Nevertheless, based on statistical analysis results and using certain generalisation, with some moderation a sign of approximate equality can be put for the precision of conversion observation obtained in respective combinations of methods.

Table 4. Comparison of distances in different methods for the internal points of test object network.

\begin{tabular}{|c|c|c|c|c|c|c|}
\hline \multirow{2}{*}{$\begin{array}{l}\text { Point number } \\
\text { from - to }\end{array}$} & \multicolumn{3}{|c|}{ Distance - D [m] } & \multicolumn{3}{|c|}{ Distance differences $-\mathrm{dD}[\mathrm{m}]$} \\
\hline & RTK & RTX & KAT. & RTK - RTX & RTK - KAT. & RTX - KAT. \\
\hline $1219-1220$ & 519.742 & 519.730 & 519.746 & 0.012 & -0.003 & -0.015 \\
\hline $1220-1221$ & 540.122 & 540.125 & 540.113 & -0.002 & 0.010 & 0.012 \\
\hline $1221-1224$ & 321.198 & 321.201 & 321.183 & -0.003 & 0.015 & 0.018 \\
\hline $1224-1225$ & 330.936 & 330.919 & 330.986 & 0.017 & -0.050 & -0.067 \\
\hline $1225-1352$ & 279.625 & 279.645 & 279.574 & -0.020 & 0.051 & 0.070 \\
\hline $1225-1228$ & 441.669 & 441.668 & 441.682 & 0.001 & -0.013 & -0.014 \\
\hline $1228-1229$ & 261.970 & 261.970 & 261.979 & 0.000 & -0.009 & -0.010 \\
\hline $1229-1230$ & 131.668 & 131.693 & 131.619 & -0.025 & 0.049 & 0.073 \\
\hline $1230-1231$ & 269.055 & 269.014 & 269.083 & 0.041 & -0.028 & -0.069 \\
\hline $1231-1232$ & 261.474 & 261.495 & 261.465 & -0.020 & 0.009 & 0.030 \\
\hline $1232-1233$ & 374.714 & 374.730 & 374.732 & -0.017 & -0.018 & -0.002 \\
\hline $1233-1234$ & 252.642 & 252.620 & 252.606 & 0.022 & 0.036 & 0.014 \\
\hline $1234-1220$ & 135.835 & 135.845 & 135.862 & -0.010 & -0.027 & -0.017 \\
\hline $1232-1243$ & 273.220 & 273.240 & 273.085 & -0.019 & 0.135 & 0.154 \\
\hline $1245-1246$ & 213.872 & 213.839 & 213.853 & 0.032 & 0.019 & -0.014 \\
\hline $1246-1247$ & 180.447 & 180.479 & 180.400 & -0.033 & 0.047 & 0.079 \\
\hline $1247-1248$ & 404.486 & 404.490 & 404.526 & -0.005 & -0.041 & -0.036 \\
\hline $1248-1249$ & 224.440 & 224.441 & 224.451 & -0.001 & -0.011 & -0.010 \\
\hline \multirow[t]{7}{*}{$1249-1229$} & 207.504 & 207.495 & 207.487 & 0.009 & 0.018 & 0.009 \\
\hline & \multicolumn{3}{|c|}{ mean value $\mu$} & -0.0010 & 0.0029 & 0.0029 \\
\hline & \multicolumn{3}{|c|}{ average standard deviation $\sigma$} & 0.0045 & 0.0072 & 0.0099 \\
\hline & \multicolumn{3}{|c|}{ test model $T$} & -0.2254 & 0.4026 & 0.2935 \\
\hline & \multicolumn{3}{|c|}{ for significance level $5 \%$ quantile $k=n-1$} & 18 & 17 & 17 \\
\hline & \multicolumn{3}{|c|}{ critical value of $T$ Student distribution } & 2.1009 & 2.1098 & 2.1098 \\
\hline & \multicolumn{3}{|c|}{ hypothesis selection } & $H(0)$ & $H(0)$ & $H(0)$ \\
\hline
\end{tabular}

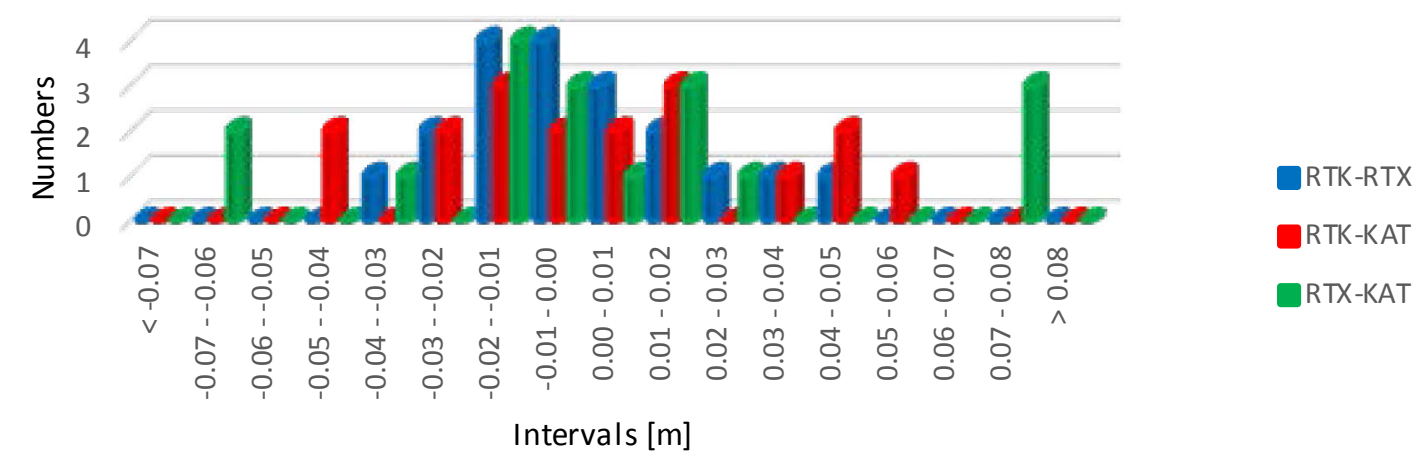

Fig. 11. Histogram of the frequency measured for distance differences $d D$ between the methods: RTK-RTX; RTK-KAT.; RTX-KAT. for internal points of test object network 
Another conversion observations subject to analysis are horizontal angles in the respective "loops" of the test object. Angles, similarly like in case of distance, have been determined based on point coordinates established in a way appropriate for each measurement method. Table 5 shows the comparison of calculated angles differences between respective methods.

Table 5. Comparison of angles in different methods for internal points of test object network.

\begin{tabular}{|c|c|c|c|c|c|c|}
\cline { 2 - 7 } \multicolumn{1}{c|}{} & \multicolumn{3}{c|}{ Angles $-\alpha$ [g] } & \multicolumn{2}{c|}{ Differences in angles - da [g] } \\
\hline Angle (L-C-P) & RTK & RTX & KAT. & $\begin{array}{c}\text { RTK - } \\
\text { RTX }\end{array}$ & $\begin{array}{c}\text { RTK }- \\
\text { KAT. }\end{array}$ & $\begin{array}{c}\text { RTX - } \\
\text { KAT. }\end{array}$ \\
\hline $1219-1220-1221$ & 175.9087 & 175.9083 & 175.9002 & 0.0004 & 0.0085 & 0.0081 \\
\hline $1234-1220-1221$ & 87.2184 & 87.2167 & 87.2376 & 0.0017 & -0.0192 & -0.0209 \\
\hline $1220-1221-1224$ & 206.0840 & 206.0777 & 206.0832 & 0.0063 & 0.0008 & -0.0055 \\
\hline $1221-1224-1225$ & 179.5085 & 179.5199 & 179.5185 & -0.0114 & -0.0100 & 0.0014 \\
\hline $1224-1225-1352$ & 187.6261 & 187.6202 & 187.6032 & 0.0059 & 0.0229 & 0.0170 \\
\hline $1224-1225-1228$ & 86.2387 & 86.2356 & 86.2245 & 0.0031 & 0.0142 & 0.0111 \\
\hline $1225-1228-1229$ & 156.6934 & 156.6932 & 156.7012 & 0.0002 & -0.0078 & -0.0080 \\
\hline $1228-1229-1230$ & 187.6535 & 187.6483 & 187.6539 & 0.0052 & -0.0004 & -0.0056 \\
\hline $1229-1230-1231$ & 204.4871 & 204.4910 & 204.4898 & -0.0039 & -0.0027 & 0.0012 \\
\hline $1230-1231-1232$ & 198.5223 & 198.5209 & 198.5170 & 0.0014 & 0.0053 & 0.0039 \\
\hline $1231-1232-1233$ & 90.5158 & 90.5176 & 90.5173 & -0.0018 & -0.0015 & 0.0003 \\
\hline $1232-1233-1234$ & 200.6783 & 200.6766 & 200.6917 & 0.0017 & -0.0134 & -0.0151 \\
\hline $1233-1234-1220$ & 202.3999 & 202.4024 & 202.3654 & -0.0025 & 0.0345 & 0.0370 \\
\hline $1231-1230-1249$ & 101.9309 & 101.9383 & 101.9195 & -0.0074 & 0.0114 & 0.0188 \\
\hline $1232-1231-1230$ & 201.4777 & 201.4791 & 201.4830 & -0.0014 & -0.0053 & -0.0039 \\
\hline $1243-1232-1231$ & 107.3409 & 107.3372 & 107.3501 & 0.0037 & -0.0092 & -0.0129 \\
\hline $1245-1243-1232$ & 193.1583 & 193.1607 & 193.1439 & -0.0024 & 0.0144 & 0.0168 \\
\hline $1246-1245-1243$ & 81.5191 & 81.5260 & 81.5137 & -0.0069 & 0.0054 & 0.0123 \\
\hline $1247-1246-1245$ & 176.3658 & 176.3504 & 176.3514 & 0.0154 & 0.0144 & -0.0010 \\
\hline $1248-1247-1246$ & 162.0047 & 162.0153 & 162.0508 & -0.0106 & -0.0461 & -0.0355 \\
\hline $1249-1248-1247$ & 209.1144 & 209.1091 & 209.0732 & 0.0053 & 0.0412 & 0.0359 \\
\hline $1230-1249-1248$ & 167.0881 & 167.0839 & 167.1144 & 0.0042 & -0.0263 & -0.0305 \\
\hline
\end{tabular}

Similarly as in case of conversion observations in relation to distance, also in the comparison of angle differences in respective combinations of methods the results are convergent. The final results included in Table 5, verifying the hypothesis, clearly determine lack of grounds for rejecting hypothesis $H_{0}$. Based on this, precision of results of conversion observations obtained from individual methods can be balanced again. However, it is worth to note certain relation between method combinations: RTK-KAT. and RTX-KAT. Although in this comparison also the range values (min.max.) are much higher than in combination RTK-RTX (from $-0.0114^{\mathrm{g}}$ to $+0.0154^{\mathrm{g}}$ ), much smaller span values, however, occur for methods RTX-KAT. (from $-0.0355^{\mathrm{g}}$ to $+0.0370^{\mathrm{g}}$ ) than in combinations RTK-KAT. (from $-0.0461^{\mathrm{g}}$ to $+0.0412^{\mathrm{g}}$ ). It is surprising in so far as Trimble RTX technology is in way a "derivative" of RTK GPS method, so at least theoretically of weaker precision parameters. In order to better illustrate the 
frequency of angle differences occurrence in the respective ranges, the results from Table 5 have been presented in form of a graph in Figure 12.

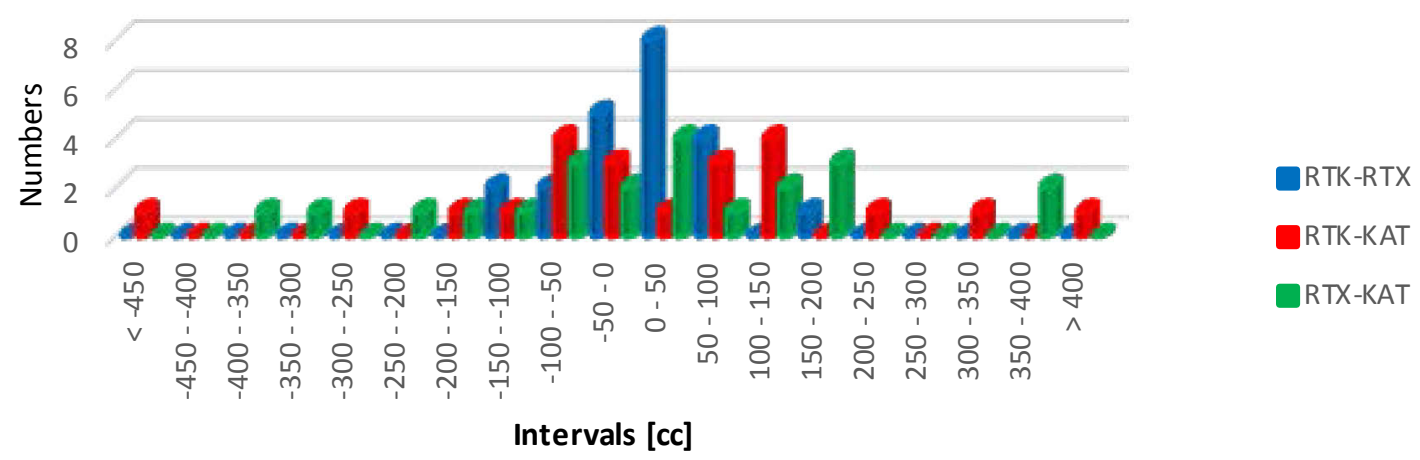

Fig. 12. Histogram of the frequency measured for angle differences da between methods: RTK-RTX; RTK-KAT.; RTX-KAT. for internal points of test object network

It is clearly visible in Figure 12 that the frequency of high differences occurrence in the compared angles is low and it only refers to combinations of methods RTK-KAT. and RTX-KAT. Much better is the precision of conversion observation in combination RTK-RTX, where the highest so called measurable frequency can be observed in the range of $0.0000^{9}$ to $+0.0050^{9}$.

The third conversion observations subject to statistical analysis in combination of individual methods are height differences between the neighbouring points of test object control network. Height differences have been calculated in analogical way (from coordinates) as previous conversion observations of distances and angles. The results of this comparison and short statistical analysis have been presented in Table 6. Also, in order to better understand comparative results obtained and conclusions that can be reached from this combination, the histogram of measured frequency has been presented in Figure 13.

Precision which can be achieved in measuring point height in RTG GPS mode is commonly known, i.e. $\pm 0.05 \mathrm{~m}$, which is very significant for the relation of the obtained conversion results. Looking at the values of height difference comparison in Table 6 and the histogram presented in Fig. 13, two conclusions can be reached. Firstly, as a result of statistical analysis test, there are no grounds for rejecting hypothesis $H_{0}$. The values of test model $T$ definitely divert from the critical values of $T$-Student distribution. Such situation was the basis for another conclusion in relation to precision evaluation of height difference conversion observations, similar like in case of distances or angles. However, the values themselves of some of the height differences in respective combinations of methods are worrying. In all three method combinations the outer values of height difference ranges are at similar level, i.e. $0.08 /-0.10 \mathrm{~m}$ do $+0.06 /+0.10 \mathrm{~m}$. Taking into consideration not only high values of the outer ranges but also the frequency of conversion observations occurrence in the established ranges (Fig. 13), precision balancing of height difference conversion observations for all comparative methods should be approached with certain scepticism. Undoubtedly, the results obtained in this manner are influenced also by the precision itself of the measurements obtained in real time. Hence the second conclusion, which recommends extra caution when it comes to generalising such precision evaluation as in case of conversion observations of distances or angles. The only optimistic conclusion that can be reached from the above analysis is certain 
convergence of the results achieved in the relation of methods RTK-RTX (the highest frequency measured within the range of $0.000 \mathrm{~m}$ to $+0.010 \mathrm{~m}$.), which in the context of the topic of this paper enables positive evaluation of Trimble RTX technology precision in relation to RTK GPS method.

Table 6. Comparison of height differences in different methods for internal points of test object network.

\begin{tabular}{|c|c|c|c|c|c|c|}
\hline \multirow{2}{*}{$\begin{array}{l}\text { Point number } \\
\text { from - to }\end{array}$} & \multicolumn{3}{|c|}{ Height differences $-\mathrm{h}[\mathrm{m}]$} & \multicolumn{3}{|c|}{ Deviations of height differences - $\mathrm{dh}[\mathrm{m}]$} \\
\hline & RTK & RTX & KAT. & RTK - RTX & RTK - KAT. & RTX - KAT. \\
\hline $1219-1220$ & 7.300 & 7.330 & 7.298 & -0.030 & 0.002 & 0.032 \\
\hline $1220-1221$ & -0.290 & -0.349 & -0.275 & 0.059 & -0.015 & -0.074 \\
\hline $1221-1224$ & -5.980 & -5.990 & -5.971 & 0.010 & -0.009 & -0.019 \\
\hline $1224-1225$ & 1.690 & 1.715 & 1.685 & -0.025 & 0.005 & 0.030 \\
\hline $1225-1352$ & -11.200 & -11.219 & -11.219 & 0.019 & 0.019 & 0.000 \\
\hline $1225-1228$ & 6.490 & 6.464 & 6.536 & 0.026 & -0.046 & -0.072 \\
\hline $1228-1229$ & 0.000 & 0.013 & 0.017 & -0.013 & -0.017 & -0.004 \\
\hline $1229-1230$ & -2.900 & -2.854 & -2.955 & -0.046 & 0.055 & 0.101 \\
\hline $1230-1231$ & -8.250 & -8.339 & -8.282 & 0.089 & 0.032 & -0.057 \\
\hline $1231-1232$ & -27.190 & -27.146 & -27.207 & -0.044 & 0.017 & 0.061 \\
\hline $1232-1233$ & 14.580 & 14.570 & 14.515 & 0.010 & 0.065 & 0.055 \\
\hline $1233-1234$ & 15.430 & 15.420 & 15.519 & 0.010 & -0.089 & -0.099 \\
\hline $1234-1220$ & 6.420 & 6.495 & 6.418 & -0.075 & 0.002 & 0.077 \\
\hline $1232-1243$ & 1.230 & 1.250 & 1.194 & -0.020 & 0.000 & 0.000 \\
\hline $1245-1246$ & 27.440 & 27.471 & 27.440 & -0.031 & 0.000 & 0.031 \\
\hline $1246-1247$ & -1.590 & -1.592 & -1.620 & 0.002 & 0.030 & 0.028 \\
\hline $1247-1248$ & 23.520 & 23.506 & 23.467 & 0.014 & 0.053 & 0.039 \\
\hline $1248-1249$ & 10.030 & 10.014 & 10.100 & 0.016 & -0.070 & -0.086 \\
\hline \multirow[t]{7}{*}{$1249-1229$} & 0.340 & 0.340 & 0.373 & 0.000 & -0.033 & -0.033 \\
\hline & \multicolumn{3}{|c|}{ mean value $\mu$} & -0.0015 & 0.0001 & 0.0006 \\
\hline & \multicolumn{3}{|c|}{ average standard deviation $\sigma$} & 0.0087 & 0.0098 & 0.0141 \\
\hline & \multicolumn{3}{|c|}{ test model $T$} & -0.1755 & 0.0057 & 0.0394 \\
\hline & \multicolumn{3}{|c|}{ for significance level $5 \%$ quantile $k=n-1$} & 18 & 17 & 17 \\
\hline & \multicolumn{3}{|c|}{ critical value of $T$ Student distribution } & 2.1009 & 2.1098 & 2.1098 \\
\hline & \multicolumn{3}{|c|}{ hypothesis selection } & $\mathrm{H}(0)$ & $\mathrm{H}(0)$ & $\mathrm{H}(0)$ \\
\hline
\end{tabular}

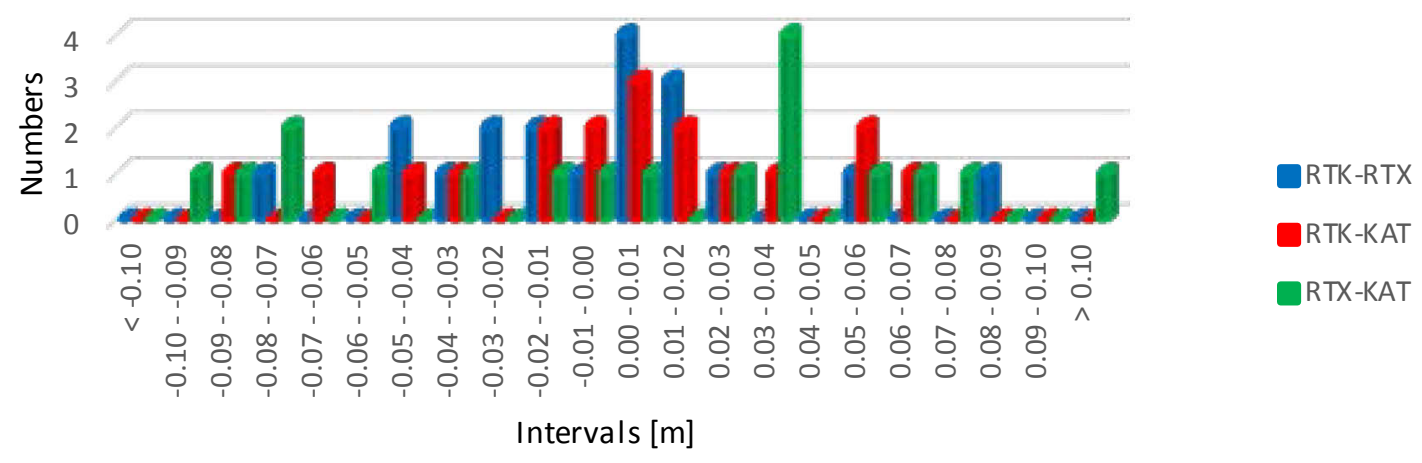

Fig. 13 Histogram of frequency measured for the deviations of height differences $d h$ between the methods: RTK-RTX; RTK-KAT.; RTX-KAT. for the points of test object network 
The next conversion observation subject to statistical analysis are control angle sums in different "loops" for respective combined methods. There are four kinds of "loops":

- loop no 1: 1220-1221-1224-1225-1228-1229-1230-1231-1232-1233-12341220 ,

- loop no 2: 1230-1231-1232-1243-1245-1246-1247-1248-1249-1230,

- loop no 3: 1352-1225-1228-1229-1230-1249-1248-1247-1352,

- loop no 4: 1219-1245-1243-1232-1233-1234-1220-1219.

For each "loop" the sum of angles determined on the basis of coordinates was calculated, and then they were compared with theoretical values to obtain angular discrepancies $k$ in respective loops. Another action was to compare the differences of discrepancies $d k$ obtained this way for each "loop". The results of comparative list along with statistical analysis are presented in Table 7.

Table 7. Comparison of angular discrepancies in different "loops" of respective methods for internal points of test object network.

\begin{tabular}{|c|c|c|c|c|c|c|}
\hline \multirow[b]{2}{*}{$\begin{array}{l}\text { Loop } \\
\text { number }\end{array}$} & \multicolumn{3}{|c|}{$\begin{array}{c}\text { Angular discrepancies in „loops” - } \mathrm{k} \\
{[\mathrm{g}]}\end{array}$} & \multicolumn{3}{|c|}{$\begin{array}{c}\text { Differences in angular discrepancies in "loops” } \\
-\mathrm{dk}[\mathrm{g}]\end{array}$} \\
\hline & RTK & RTX & KAT. & RTK - RTX & RTK - KAT. & RTX - KAT. \\
\hline 1 & -0.0001 & -0.0001 & 0.0001 & 0.0000 & -0.0002 & -0.0002 \\
\hline 2 & -0.0001 & 0.0000 & 0.0000 & -0.0001 & -0.0001 & 0.0000 \\
\hline 3 & 0.0001 & -0.0001 & 0.0000 & 0.0002 & 0.0001 & -0.0001 \\
\hline \multirow[t]{7}{*}{4} & 0.0001 & 0.0001 & -0.0001 & 0.0000 & 0.0002 & 0.0002 \\
\hline & \multicolumn{3}{|c|}{ mean value $\mu$} & 0.0000 & 0.0000 & 0.0000 \\
\hline & \multirow{2}{*}{\multicolumn{3}{|c|}{ average standard deviation $\sigma$}} & 0.0001 & 0.0001 & 0.0001 \\
\hline & \multicolumn{2}{|c|}{ test model $T$} & & 0.3974 & 0.0000 & -0.2928 \\
\hline & \multicolumn{3}{|c|}{$\begin{array}{l}\text { for significance level } 5 \% \text { quantile } \\
k=n-1\end{array}$} & 3 & 3 & 3 \\
\hline & \multicolumn{3}{|c|}{$\begin{array}{l}\text { critical value of } T \text { Student } \\
\text { distribution }\end{array}$} & 2.7764 & 2.7764 & 2.7764 \\
\hline & \multicolumn{3}{|c|}{ hypothesis selection } & $\mathrm{H}(0)$ & $H(0)$ & $\mathrm{H}(0)$ \\
\hline
\end{tabular}

Both the results included in Table 7 and their visualization in Fig. 14 definitely support balancing the precision of conversion observation in the context of control sums calculated in respective "loops". It is confirmed not only by lack of grounds for rejecting the hypothesis $H_{0}$ (Table 7 ), but also by the values themselves of these discrepancies in respective combinations of methods.

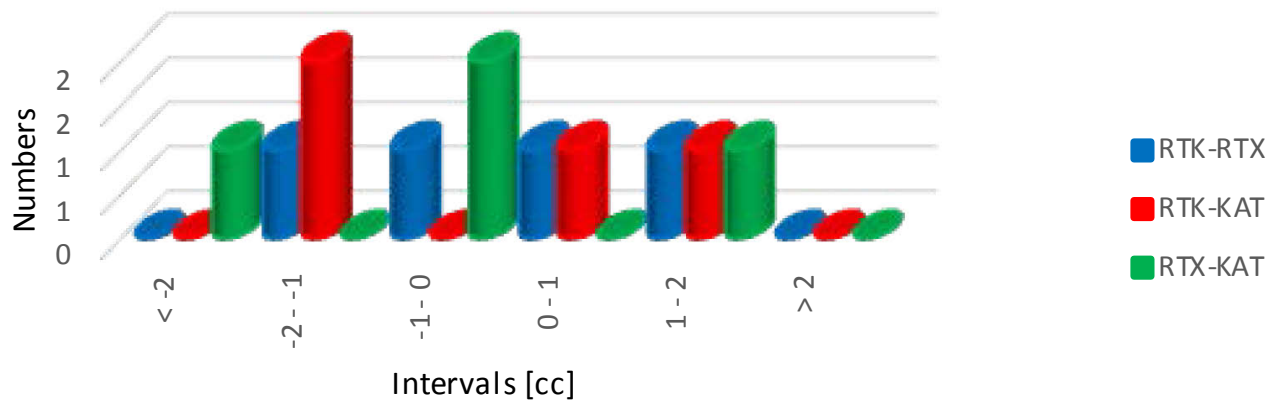

Fig. 14. Histogram of frequency measured for the differences of angular discrepancies $d k$ in "loops" between methods: RTK-RTX; RTK-KAT.; RTX-KAT. for the points of test object network 
The last conversion observations subject to statistical analysis are area fields in the above-mentioned "loops". The comparison results have been presented in Table 8 and illustrated in Fig. 15. Similarly to previous conclusion (in relation to control sums), also in this case practically unnoticeable differences in area fields can be seen between the measurement methods in different combinations. In combinations of RTK-RTX measurement modes the two loops do not present almost any differences, and other stay at almost zero level (merely few metres). Also in other cases area fields differ in merely a fraction of a percentage. Another indicator for equal conversion observation precisions of area fields is the conclusion that leads to lack of grounds for rejecting the hypothesis $H_{0}$.

Table 8. Comparison of area fields differences in different "loops" of respective methods for internal points of test object network.

\begin{tabular}{|c|c|c|c|c|c|c|}
\hline \multirow{2}{*}{ Loop number } & \multicolumn{3}{|c|}{ Area fields [M2] - P } & \multicolumn{3}{|c|}{ Differences in area fields [M2] - dP } \\
\hline & RTK & RTX & KAT. & RTK - RTX & RTK - KAT. & RTX - KAT. \\
\hline 1 & 623945 & 623945 & 623941 & 0 & 5 & 4 \\
\hline 2 & 389905 & 389905 & 389926 & 0 & -22 & -22 \\
\hline 3 & 345588 & 345593 & 345539 & -5 & 50 & 54 \\
\hline \multirow[t]{7}{*}{4} & 390595 & 390589 & 390583 & 6 & 12 & 7 \\
\hline & \multicolumn{3}{|c|}{ mean value $\mu$} & 0 & 11 & 11 \\
\hline & \multicolumn{3}{|c|}{ average standard deviation $\sigma$} & 2 & 15 & 16 \\
\hline & \multicolumn{3}{|c|}{ test model $T$} & 0.0987 & 0.7608 & 0.6931 \\
\hline & \multicolumn{3}{|c|}{ for significance level $5 \%$ quantile $k=n-1$} & 3 & 3 & 3 \\
\hline & \multicolumn{3}{|c|}{ critical value of $T$ Student distribution } & 2.7764 & 2.7764 & 2.7764 \\
\hline & \multicolumn{3}{|c|}{ hypothesis selection } & $H(0)$ & $\mathrm{H}(0)$ & $H(0)$ \\
\hline
\end{tabular}

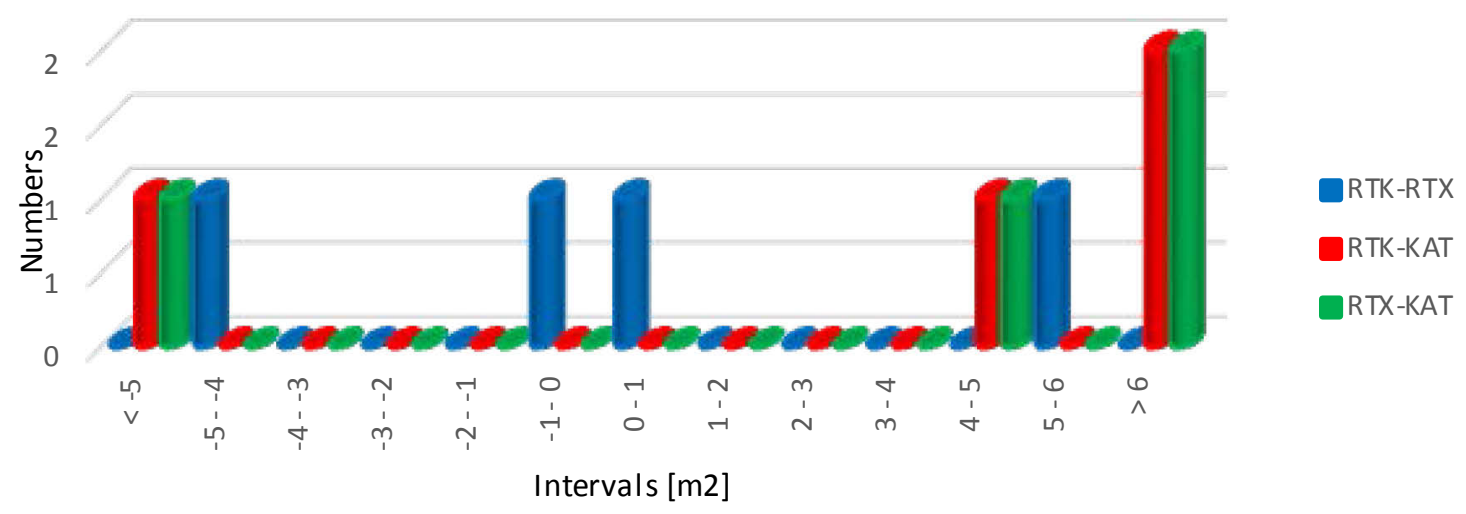

Fig. 15. Histogram of frequency measured for the differences in area fields $d P$ in "loops" between methods: RTK-RTX; RTK-KAT.; RTX-KAT. for the points of test object network

Based on the presented Tables 1-8 and Figures 7-15 the precision of measurements obtained in RTX mode can be verified in the context of conversion observations in relation to parameters established as reference basis.

\section{Conclusions}

Conclusion with regards to analysis results of conversion observations in method combinations RTK-RTX, RTK-KAT. or RTX-KAT. is complex. Statistical analyses related to study results lead to conclusions which are seemingly conflicting. This divergence is especially visible in the verification of research sample homogeneity 
(research in relation to KRA1 station) and the conclusions reached on this basis, and then referring them to the results of statistical analyses for the test object in a small area (research in relation to internal points of the test network). In the first case, for the evaluated conversion observations - increments of horizontal coordinates, distances and elevations - Trimble RTX technology with xFill function can be acknowledged an excellent solution, enabling continuation of measurements in RTK mode without losing significant precision parameters. On the other hand, referring these real time measurements - RTK and RTX - to values which are a conversion of detailed control network point coordinates exceed the precision criteria. This conclusion confirms study results included in (Krzyżek, 2013) in relation to absolute parameters. Of slightly different nature are the conclusions reached from statistical analysis in second case, i.e. when the same points of the test network were subject to study, however, to the exclusion of KRA1 station. In this study version, all relations of respective combinations of methods (RTK-RTX, RTK-KAT., RTX-KAT.) were convergent, i.e. maintained study results at similar precision level.

The difference between two cases of analysis referred, among others, to size of the test object. In relation to test points for a permanent station the object area was at the level of approx. 1.5 thousand hectares, while for the relation of internal network points the area of the field was about 40 hectares. As it was mentioned before, the study sample was not fully homogeneous. The difference between conclusions in two cases of statistical analysis is probably related to scale parameter. For both objects of conversion observations, translation or rotation parameters are probably of little significance. For the parameter of scale, the area which is about 40 times larger (first case of the object) than the area of the analysed object in the second case is definitely affected by the scale parameter. Therefore, different conclusions were defined (between both cases) on the basis of statistical analysis in the context of method relations RTK-KAT. and RTX-KAT.

The conclusions reached from the study so far allow to verify the precision and application possibilities of conversion observations obtained thanks to Trimble RTX technology. It is a real time measurement method which can be freely and with full justification used for carrying out every day geodetic tasks. It can be applied both in order to obtain absolute values and relative parameters. However, the relation of measured or conversion values, obtained in RTX mode, towards reference values should always be taken into consideration. Being aware of that will allow to verify the possibility of Trimble RTX technology application in geodetic surveying.

\section{Acknowledgments}

This work was carried out within the statutory studies of the AGH University of Science and Technology, Faculty of Mining Surveying and Environmental Engineering No. 11.11.150.006.

\section{References}

Bosy J., Kontny B., \& Borkowski A. (2009). International Association of Geodesy Symposia Volume 134, Geodetic Reference Frames, IAG Symposium Munich Germany, October 9-14, 2006, pp197-203, Copyright 2009, Springer.

Dai L., Wang J., Rizos C., \& Han S. (2003). Predicting atmospheric biases for realtime ambiguity resolution in GPS/GLONASS reference station networks. Journal of Geodesy, Vol. 76, Issue 11-12, 617-628. 
Geng J., Meng X., Dodson A., Ge M., \& Teferle F. (2010). Rapid re-convergences to ambiguity-fixed solution in precise point positioning, Journal of Geodesy, Vol. 84, Issue $12,705-714$.

Krzyżek R. (2013). Verification of applicability of the Trimble RTX satellite technology with xFill function in establishing surveying control networks, Geodesy and Cartography, Vol. 62, no. 2, s. 217-233.

Kubo N. (2009). Advantage of velocity measurements on instantaneous RTK positioning. GPS Solution, Vol. 13, Issue 4, 271-280.

Lannes A., \& Prieur J-L. (2013). Calibration of the clock-phase biases of GNSS networks: the closure-ambiguity approach. Journal of Geodesy, Vol. 87, Issue 8, 709-731.

Leonardo R., Landau H., Nitschke M., Glocker M., Seeger S., Chen X., Deking A., Ben Tahar M., Zhang F., Ferguson K., Stolz R., Talbot M., Lu G., Allison T., Brandl M., Gomez V., Cao W., Kipka A., \& Trimble Terrasat GmbH, Germany. (2011). RTX Positioning: The next generation of cm-accurate Real-Time GNSS Positioning. White Paper_RTX.

MIA. (2011). Regulation of Minister of Interior and Administration - in case of technical standards of performing detailed surveys and working out and sending results of these surveys to National Geodetic and Cartographic Database (in Polish). Journal of Laws of 2011 No. 263, entry 1572. Warsaw: Government Legislation Centre.

Zhang X., Li X., \& Guo F. (2011). Satellite clock estimation at $1 \mathrm{~Hz}$ for realtime kinematic PPP applications. GPS Solutions, Vol. 15, Issue 4, 315-324.

Zhang X., \& Li P. (2013). Integrating GPS and GLONASS to accelerate convergence and initialization times of precise point positioning. GPS Solution.

www.asgeupos.pl - the state of the 30.11 .2013

\section{Authors:}

Robert Krzyżek ${ }^{1)}$, rkrzyzek@agh.edu.pl

1) AGH University of Science and Technology, Faculty of Mining Surveying and Environmental Engineering, Department of Geomatics, 30 Mickiewicza Al., 30-059 Krakow, Poland 